\title{
Impact of A Peer-Led, Community-Based Parenting Programme Delivered At A National Scale: An Uncontrolled Cohort Design With Benchmarking
}

Crispin Day ( $\square$ crispin.1.day@kcl.ac.uk)

South London and Maudsley NHS Foundation Trust

Joshua Harwood

Harwood Child Psychology

Nadine Kendall

King's College London

Jo Nicoll

South London and Maudsley NHS Foundation Trust

Research Article

Keywords:

Posted Date: February 9th, 2022

DOI: https://doi.org/10.21203/rs.3.rs-1180221/v1

License: (c) (i) This work is licensed under a Creative Commons Attribution 4.0 International License.

Read Full License 
2 Impact of a peer-led, community-based parenting programme delivered at a national scale: an uncontrolled cohort design with benchmarking

4

5

6

7

8

9 Corresponding Author: Crispin Day, Centre for Parent and Child Support, South London

10 and Maudsley NHS Foundation Trust, Michael Rutter Centre, De Crespigny Park,

11 Camberwell, London SE5 8AZ.

12 Email: crispin.1.day@kcl.ac.uk

13

14 Joshua Harwood, Harwood Child Psychology, London.

15

16 Nadine Kendall, King's College London, Department of Psychology, Institute of Psychiatry

17 Psychology \& Neuroscience.

18

19 Jo Nicoll, South London and Maudsley NHS Foundation Trust, London.

20

21

22

23

Abstract 
2 Background

3 Childhood behavioural are the most common mental health disorder worldwide and represent

4 a major public health concern, particularly in socially disadvantaged communities. Treatment

5 barriers mean that up to $70 \%$ of children do not receive recommended parenting

6 interventions. Innovative approaches, including evidence-based peer-led models, such as

7 Empowering Parents Empowering Communities' (EPEC) Being a Parent (BAP) programme,

8 have the potential to reduce childhood difficulties and improve parenting if replicable and

9 successfully delivered at scale.

11 Method

12 This real-world quasi-experimental study, with embedded RCT benchmarking, examined the

13 population reach, attendance, acceptability and outcomes of 128 BAP groups ( $n=930$ parents)

14 delivered by 15 newly established sites participating in a UK EPEC scaling programme.

16 Results

17 Scaling programme (SP) sites successfully reached parents living in areas of greater social

18 deprivation ( $\mathrm{n}=476,75.3 \%)$, experiencing significant disadvantage ( $45.0 \%$ left school by 16 ;

$1939.9 \%$ lived in rental accommodation; 36.9\% lone parents). The only benchmarked

20 demographic difference was ethnicity, reflecting the greater proportion of White British

21 parents living in scaling site areas (SP 67.9\%; RCT 22.4\%). Benchmark comparisons showed

22 scaling sites' parent group leaders achieved similar levels of satisfaction. Scaling site parent

23 participants reported substantial levels of improvement in child concerns (ES 0.6), parenting

24 (ES 0.9), parenting goals (ES 1.2) and parent wellbeing (ES 0.6) that were of similar 
1 magnitude to RCT benchmarked results. Though large, parents reported lower levels of

2 parenting knowledge and confidence acquisition compared with the RCT benchmark.

3

4 Conclusion

5 Despite common methodological limitations associated with real-world scaling evaluations,

6 findings suggest that this peer-led, community-based, parenting approaches can be replicated

7 at scale and may have considerable potential to improve child and parenting difficulties,

8 particularly for socially disadvantaged populations.

9

10

11

12

13

14

15

16

17

18

19

20

21

22

23 


\section{Background}

3 Childhood behavioural disorders, characterised by persistent aggressive, oppositional and

4 defiant behaviours, are the most common mental health disorder worldwide, representing a

5 growing public health concern with poor outcomes persisting into adulthood [1-5]. In the

6 UK, 4.6\% of children aged 5-19 years meet behaviour disorder criteria [6], and a further 15-

$7 \quad 20 \%$ have significant, subclinical difficulties. UK lifetime estimated costs range from

$8 £ 85,000$ per moderate case to $£ 260,000$ per severe case [7]. Behaviour disorders are twice as

9 common in disadvantaged neighbourhoods and communities, and between two to four times

10 more likely in families living in poverty, receipt of disability and other welfare benefits [7].

12 Up to $70 \%$ of children with behavioural disorders do not receive recommended interventions

13 [8]. Barriers to care include insufficient service capacity, limited availability of evidence-

14 based intervention, complex access arrangements, long waiting times, family stigma, and

15 poor lay mental health knowledge. Typically, interventions are offered by highly trained

16 specialist mental health professionals with postgraduate level education, delivered at clinic

17 and healthcare facilities using evidence-informed rather than manualised, evidence-based

18 interventions. These barriers hinder problem identification, parent help-seeking, and limit the

19 capability of routine services to deliver effective care at sufficient scale to substantially

20 reduce prevalence and impact, particularly for low income and Minoritised families $[9,10]$

22 Group-format evidence-based parenting approaches are effective when tested in highly

23 controlled experimental trial conditions and recommended as the first line response [11-13].

24 These approaches can maintain performance under in real world conditions but cost and 
1 almost exclusive dependence on delivery by highly trained and specialist practitioners inhibit

2 availability at the scale required [14-17].

4 There is significant concern about the continuing failure to meet child and family need. The

5 use of more innovative approaches, including peer-led models, has been recommended [18].

6 Less is known about the delivery at scale of peer-led approaches [19,20], in which

7 manualised parenting approaches are delivered by trained and quality assured non-

8 professionals with the aim of increasing access, acceptability and reach, particularly for low

9 income and Minoritised families. If effective at scale, lower associated service costs of these

10 approaches can potentially increase capacity and reduce the treatment gap.

12 Benchmarking is potentially an efficient, low-cost method that can be used to systematically

13 examine the performance of evidence-based approaches as they travel from definitive and

14 pragmatic trials to novel settings and real-world conditions [21-23]. Benchmarking can not

15 only compare outcomes but can also assess target population reach and acceptability. As a

16 relatively novel approach, benchmarking has been used to assess interventions in acute

17 medicine and adult mental health but rarely in the field of child mental health and parenting.

19 Scaling-up and scaling-out evidence-based approaches

20 Real world replication is complicated, unpredictable and success is not guaranteed [24,25].

21 Scaling-up involves dissemination based on established conditions in which new providers typically adhere to pre-determined methods and protocols intended to reproduce trial outcomes. Pre-determined trial conditions may be challenging to reproduce in real world

24 settings. Scaling-out, on the other hand, refers to replication in conditions that differ from 
1 original trial conditions [24], potentially offering great flexibility but risking variations in

2 population reach, delivery, and fidelity that can undermine performance $[25,26]$

4 Empowering Parents Empowering Communities Scaling Programme

5 Empowering Parents Empowering Communities (EPEC) is a task sharing, peer-led parenting

6 approach. Its' group-based parenting course format is consistent with policy

7 recommendations and intended to build social support between participants, optimise impact,

8 and lower unit cost. EPEC is delivered in local, community locations and the programme

9 uses high visibility, pro-active local outreach campaigns to engage parents. Within these

10 targeted community locations, an open access approach is typically used, rather than formal

11 referral. The peer-led format is associated with high levels of parent engagement, acceptability and reduced stigma. Randomised control trial and field evidence shows that EPEC Being a Parent successfully reaches socially disadvantaged and Minoritised parents of children aged 2-11 years, is highly acceptable, and produces significant improvements in child behaviour, positive parenting and parental concerns when delivered by peer parent group leaders (PGLs) recruited from within target populations, directly trained and supervised by EPEC developers [19,20].

Funded by the UK Early Years Social Action Fund, NESTA and Department for Culture,

20 Media and Sport, the EPEC Scaling Programme examined the scalability of the Being a

21 Parent parenting course in 15 newly established EPEC Hubs located in socially disadvantaged areas across England for parents of children aged 2-5-years. The Programme scaled-up established EPEC methods, including its peer-led approach, manualised training, quality assurance procedures, and scaled-out by testing delivery in new service organisations types, such as local authorities and voluntary organisations, rural as well as new urban 
1 settings, inclusion of socially disadvantaged populations that potentially differed in

2 characteristics from previous research and field trials, and novel hub setup, parent group

3 leader recruitment, and implementation support methods.

5 The evaluation reported in this paper had two aims:

6 1. To examine the parent population reach, parent attendance and acceptability across Scaling Programme sites and compare these with established Being a Parent RCT benchmarks.

2. To evaluate the impact of the Being a Parent parenting course across Scaling Programme sites on child, parent and parenting outcomes and compare these with established RCT benchmarks.

\section{Method}

14 Design

15 A pragmatic cohort design incorporating a benchmarking comparison derived from 16 previously published RCT results was used [19]. Demographic information and outcome

17 measures were collected at the beginning (Time 1) and end (Time 2) of the Being a Parent

18 parenting course, acceptability data were collected at Time 2. Attendance data were collected

19 throughout each parenting course. Over the course of the evaluation period, 1135 parents

20 attended a Being Parent information session, $930(89.9 \%)$ of these participated in the

21 parenting course, of whom 730 (78.4\%) completed Time 1 measures and $405(55.5 \%)$

22 completed Time 2 measures.

\section{Participants}


1 New EPEC Hubs

2 Fifteen EPEC hub host organisations: 10 local authorities, three NHS Trusts and two

3 charitable organisations were selected because of compatibility between their local strategic

4 priorities, operational resources, parenting and peer expertise, and population needs, and

5 EPEC aims and programme theory, see Appendix 1. Over the 18-month duration of the

6 Scaling Programme, hubs delivered 128 Being a Parent parenting groups from 97 different

7 venues. Sixty-five venues (67.0\%) were in the lowest third of the most deprived UK

8 neighbourhoods, with 29 venues (29.9\%) in the $10 \%$ most deprived areas [27].

9

10 Participant parents

11 Parents were eligible for the Being a Parent course when they were a primary parental caregiver who: 1) reported difficulties in managing behaviour of an index child aged 2-5

13 years, and 2) expressed concerns about their parenting. Families were excluded when the

14 parent: 1) had insufficient English to complete evaluation measures, 2) could not attend

15 weekly course sessions, 3) was not living with the index child, and 4) the child experienced

16 significant neurodevelopmental difficulties, such as autism.

18 Measures

19 Demographic information: Included parent age, ethnicity, first language, parent status,

20 educational qualifications, housing and employment status.

Clinical outcomes

23 In families with more than one child aged 2-5 years, participants completed measures on the

24 child about whom they had most significant concerns. 
1 Concerns About My Child (CAMC, 19). An idiographic measure of parental perception of

2 child difficulties, previously used in Being a Parent trial evaluation. Parents rate up to three

3 main child emotional and behavioural concerns from 0 (not concerned at all) to 100 (could

4 not be more concerned). Concerns were categorised into five domains: Conduct Problems,

5 Parent-Child Relationship and Communication Difficulties, Self-Regulation, Emotional

6 Distress and Other.

7 Arnold O'Leary Parenting Scale (PS, 28). Previously used in the Being a Parent trial, this

8 30-item questionnaire assesses dysfunctional parental discipline styles for children aged 2-16

9 years, yields a total score and parental verbosity, over-reactivity and laxness subscales.

10 Lower scores indicate more positive parenting skills. Total score $\geq 3.2$ differentiates between

11 clinic and non-referred children. In this study, there was good internal consistency for the

12 total score $(\alpha=.77)$.

13 My Parenting Goals (MPG). An idiographic measure of up to two personal parenting goals,

14 using a visual analogue scale from 0 (could not be further from achieving my goal) to 100

15 (goal completely achieved).

16 Short Warwick Edinburgh Mental Wellbeing Scale (SWEMWBS, 29). A seven-item

17 parent wellbeing measure each rated on a 5-point Likert scale. High scores represent greater

18 mental wellbeing. SWEMWBS is sensitive to change and the full version has been used in

19 evaluations of parenting programmes. It had good internal consistency, $\alpha=.85$. Raw

20 SWEMWBS scores were transformed to allow comparisons with national survey data.

Being a Parent acceptability and satisfaction

23 Treatment Acceptability Rating Scale (TARS - 19). This 12-item self-report questionnaire,

24 previously used in Being a Parent trial, uses a 4-point Likert scale to assess, (i) parenting

25 knowledge, skills and confidence acquired (TARS KSC - 4 items yield total score 4-16) and 
1 (ii) course satisfaction and quality (TARS SQ - 5 items yield total score 5-20). Higher scores

2 indicate greater acceptability and satisfaction. Three free-text items cover helpful and

3 unhelpful participant experience.

4

5 Being a Parent attendance

6 Parent attendance, non-attendance, cancellation and drop-out was recorded prospectively by

7 parent group leaders for each Being a Parent course using a secure online spreadsheet that

8 generated an anonymised identifier for each parent.

10 EPEC Being a Parent Scaling Programme

11 This comprised three inter-related phases:

12 Phase 1: Hub engagement and initial set up (0-6 months): Hub site selection, licence

13 agreement, staff appointment, initial 3-day hub familiarisation training covering Being a

14 Parent quality standards and functions, staff roles and responsibilities, and evaluation.

16 Phase 2: Hubs organisation (3-9months): Hub staff training in Being a Parent manualised

17 content and methods (4-days), PGL recruitment and training, supervision and quality

18 assurance (3-days), and engagement of local stakeholders and communities. Each hub used

19 existing local family, service and community networks to recruit an initial cohort of 12-16

20 PGLs, who completed a certified 60-hour training covering: (1) Being a Parent knowledge,

21 methods and skills, (2) child development and family resilience, (3) group dynamics and

22 facilitation skills, and (4) local safeguarding procedures. Participants completed an assessed

23 portfolio and supervised practice prior to certification. 
1 Phase 3: Hub implementation (6-18 months): Each hub established pathways to engage

2 local parents, ran 'coffee morning' information sessions, organised a rolling programme of

3 supervised Being a Parent groups. National EPEC consultants used manualised quality

4 standards to appraise hub implementation, problem-solve and support site scaling using

5 ongoing digital and face-to-face contact and quarterly collaborative Hub learning and

6 exchange events equivalent to one-day per month.

7

8 Findings are available elsewhere that describe the acceptability and impact of the training

9 provided to hubs during the Scaling Programme and the demographic characteristics and

10 training outcomes for parent group leaders recruited by hubs [30]. Working in pairs, 159

11 certified parent group leaders delivered 128 Being a Parent courses, each co-delivering one

12 to four groups.

14 Being a Parent Course

15 The Being a Parent course consisted of eight, two-hour sessions, with on-site crèche

16 facilities, for 8-12 parents. It used large and small group discussion, information sharing,

17 demonstrations, practice and homework to enable parents to acquire key parenting

18 knowledge, understanding and skills based on child development, social learning, attachment,

19 systems, family relations, communication and reflective function concepts. This content

20 covered parent wellbeing and expectations; understanding children's needs, emotions and

21 behaviour; child-led play, listening and communication; praise and encouragement; and

22 positive discipline strategies. Course completion was based on attendance of five or more

23 sessions [19]. 
1 Participants were recruited through direct parent contact, word of mouth, recommendation by

2 existing community and specialist services, and printed information and posters available in

3 key family community locations, such as children's centres and local schools. Prior to

4 enrolment, prospective parents were invited to an introductory 'coffee morning' information

5 session. Course fidelity and quality assurance, designed to monitor and maintain course

6 norms, consolidate PGL skills, provide support and monitor safety, was undertaken through

7 1) PGL fortnightly supervision and (2) supervisor fortnightly observation of course delivery

8 and practice.

9

10 Procedure

11 After registration and prior to the first course session, participant parents received a link to a secure online Qualtrics survey portal to confirm consent and complete Time 1 measures using

13 a uniquely generated anonymised identifier. Time 2 data was collected via a second

14 Qualtrics link sent prior to the final course session. Online data was returned digitally

15 directly to the Scaling Programme evaluation team at King's College London. Parents could

16 withdraw from the evaluation without it affecting their participation in the parenting course.

17 The study team did not have the resources to follow-up parents who did not complete Time 2 18 measures.

\section{Service evaluation and informed consent}

21 The aims of this evaluation met criteria for service evaluation rather than research or audit

$22[31,32]$. It was designed and conducted with the sole purpose of defining or judging the 23 service provided by the national EPEC dissemination team. The service evaluation did not 24 explore nor seek to undertake an experiment to investigate or establish broader evidence 25 about wider research issues related to parenting interventions nor implementation science. 
2 Each parent participating in the service evaluation provided consent prior to completing the

3 evaluation measures. Data was anonymised using individual parent codes. The service

4 received by the parents was not conditional nor affected by taking part in the evaluation.

6 Analysis plan

7 A cohort analysis using a merged dataset from across participating sites was conducted. An

8 intention to treat analysis was not planned because of the increased likelihood of substantial

9 data loss in large scale community evaluations of this type [15,33]. No between site

10 comparisons were planned due to the limited sample sizes available for individual sites.

11 Statistical analysis was mainly descriptive using means and SD for continuous demographic, acceptability and attendance data, and medians and range for skewed data. Frequencies and proportions were used to describe categorical variables. Continuous variables were compared using independent sample t-tests and proportion variables were compared using chi squared analysis. Clinical outcome change scores (Time 2 minus Time 1) were calculated for

16 all measures and t-tests. Cohen's d effect sizes $(a=0.05)$ were calculated as follows:

17 Cohen's $\mathrm{d}_{\mathrm{av}}=\frac{\overline{x 1}-\overline{x 2}}{\sqrt{\frac{S D_{1} 1^{2}+S D_{2}^{2}}{2}}}$

18 To reduce potential bias, univariate outliers were removed pairwise when any data point that 19 was $z=+/-3.29$ from the paired sample mean difference score, resulting in the removal of two 20 cases $[34,35]$.

22 An established benchmarking methodology was used to compare CAMC and PS outcomes 23 with the RCT comparison. Effect sizes were calculated using the same formula for paired 24 samples and standardised for comparison between the two samples, with the use of noncentral t-tests and confidence intervals set to $95 \%[36,37]$. The non-central distribution was 
1 used to take account of the differences of power in the calculation of effect sizes according to

2 sample size. It was assumed that standardised effect size values with non-overlapping

3 confidence intervals were indicative of significant differences between the scaling and

4 benchmark samples [38]. An effect size difference of $d=0.2$ was considered to be clinically

5 meaningful [39].

6

7 Analyses showed little systematic bias between participants providing data at both time

8 points and those only completing Time 1 measures (see Appendix 2: Tables 1 and 2). Parents

9 included in the analysis only differed by Time 1 CAMC scores and were more likely to be

10 White British.

12 Results

14 Being a Parent reach, attendance and acceptability

15 The mean age for parents was 34.3 years, with $53.3 \%$ aged between 28 - 38 years and $20.3 \%$

16 aged between 21-27 years, see Table 1 . The majority of parents were mothers $(92.3 \%)$ and

17 White British (67.9\%). The largest minority group was South Asian (10.7\%).

19 The majority of parents $(n=476,75.3 \%)$ lived in areas with higher than UK average social

20 deprivation. Over a quarter $(27.4 \%, \mathrm{n}=173)$ lived in communities categorised in the $10 \%$

21 most socially deprived (33), 45.0\% left school by age $16,39.9 \%$ lived in rental

22 accommodation, $21.4 \%$ were involuntarily unemployed and $36.9 \%$ were lone parents, see

23 Table 1. 
1 The only demographic differences between scaling and benchmark samples were ethnicity

2 and first language, with a greater proportion of White British parents (Scaling

3 sample $=67.9 \%$; RCT sample $\left.=22.4 \%, \chi^{2}(1)=48.25, \mathrm{p}<0.05\right)$ and lower proportion of parents

4 with English as a second language (Scaling sample=23.9\%; RCT sample $=46.6 \%$,

$\left.5 \quad \chi^{2}(1)=14.31, \mathrm{p}<0.05\right)$, see Table 1 .

6

7 Table 1: Comparison of Scaling Programme and Being a Parent RCT parent demographic

8 characteristics

\begin{tabular}{|l|l|c|c|c|c|c|}
\hline \multirow{2}{*}{\begin{tabular}{l}
\multirow{2}{*}{ chemographic } \\
characteristic
\end{tabular}} & \multirow{2}{*}{ Value } & \multicolumn{2}{|c|}{ Pcaling } & \multicolumn{2}{c|}{ RCT } & Sig \\
\cline { 3 - 7 } & & $\mathrm{N}$ & $\%$ & $\mathrm{~N}$ & $\%$ & $\mathrm{p}$ \\
\hline Parent gender & Female & 648 & 92.3 & 56 & 96.6 & n.s \\
\hline Parent ethnicity & White British & 452 & 67.9 & 13 & 22.4 & $<0.05$ \\
\hline English as a second & Yes & 159 & 23.9 & 27 & 46.6 & $<0.05$ \\
\hline language & & & & & & \\
\hline Lone parent status & Yes & 244 & 36.9 & N/A & N/A & N/A \\
\hline Parents highest & University education & 155 & 23.8 & 20 & 36.2 & n.s \\
\hline Tyalification & completed & & & & & \\
\hline Work status & Unemployed & 141 & 21.4 & 12 & 22.4 & n.s \\
\hline
\end{tabular}

9

10 Being a Parent Attendance

11 Six hundred and eighty-four parents (73.5\%) completed the BaP course across the 15 sites.

12 This was significantly less than the $92.0 \%$ course completion rate achieved in the Being a

13 Parent $\operatorname{RCT}\left(\chi^{2}(1)=9.47, \mathrm{p}<0.01\right)$. 
Being a Parent Acceptability

3 Parents reported high levels of satisfaction with course quality (TARS SQ), see Table 2.

4 Scaling sample TARS SQ ratings were not significantly different from the benchmark

5 comparison (Mean Scaling TARS SQ score=18.8 ( $\mathrm{SD}=1.8)$, Mean RCT, TARS SQ

$6 \quad$ score $=18.9,(\mathrm{SD}=1.4), \mathrm{t}(453)=0.4, \mathrm{p}=\mathrm{ns})$.

7

8

9

10

11

12

13

14

15

16

17

18

19

20

21

22

23 Table 2: Scaling Programme TARS Course Satisfaction and Quality Results 


\begin{tabular}{|c|c|c|c|c|}
\hline Treatment Acceptability Rating Scale & Not at all & A little & Quite a lot & $\begin{array}{l}\text { A great } \\
\text { deal }\end{array}$ \\
\hline \multicolumn{5}{|l|}{ Course Satisfaction \& Quality } \\
\hline $\begin{array}{l}\text { Being a Parent group leader } \\
\text { competence }\end{array}$ & $\begin{array}{l}0 \% \\
(\mathrm{n}=0)\end{array}$ & $\begin{array}{l}1.2 \% \\
(\mathrm{n}=5)\end{array}$ & $\begin{array}{l}20.5 \% \\
(n=83)\end{array}$ & $\begin{array}{l}78.2 \% \\
(n=318)\end{array}$ \\
\hline $\begin{array}{l}\text { Overall satisfaction with Being a } \\
\text { Parent course }\end{array}$ & $\begin{array}{l}0.2 \% \\
(\mathrm{n}=1)\end{array}$ & $\begin{array}{l}0.7 \% \\
(n=3)\end{array}$ & $\begin{array}{l}29.3 \% \\
(n=119)\end{array}$ & $\begin{array}{l}69.7 \% \\
(n=283)\end{array}$ \\
\hline $\begin{array}{l}\text { Being a Parent covered appropriate } \\
\text { content/topics }\end{array}$ & $\begin{array}{l}0 \% \\
(n=0)\end{array}$ & $\begin{array}{l}2.2 \% \\
(n=9)\end{array}$ & $\begin{array}{l}24.2 \% \\
(n=98)\end{array}$ & $\begin{array}{l}73.6 \% \\
(n=299)\end{array}$ \\
\hline $\begin{array}{l}\text { Being a Parent group leaders } \\
\text { communicated effectively }\end{array}$ & $\begin{array}{l}0 \% \\
(\mathrm{n}=0)\end{array}$ & $\begin{array}{l}0.5 \% \\
(\mathrm{n}=2)\end{array}$ & $\begin{array}{l}18.5 \% \\
(n=75)\end{array}$ & $\begin{array}{c}81 \% \\
(n=329)\end{array}$ \\
\hline $\begin{array}{l}\text { Being A Parent group leaders were } \\
\text { motivating (e.g., energetic, attentive) }\end{array}$ & $\begin{array}{c}0 \% \\
(\mathrm{n}=0)\end{array}$ & $\begin{array}{l}1.5 \% \\
(n=6)\end{array}$ & $\begin{array}{l}14.3 \% \\
(n=58)\end{array}$ & $\begin{array}{l}84.2 \% \\
(n=342)\end{array}$ \\
\hline \multicolumn{5}{|l|}{ TARS Knowledge, Skills and Confidence } \\
\hline $\begin{array}{l}\text { Improved understanding of positive } \\
\text { parenting }\end{array}$ & $\begin{array}{l}0 \% \\
(\mathrm{n}=0)\end{array}$ & $\begin{array}{l}5.2 \% \\
(n=21)\end{array}$ & $\begin{array}{c}40 \% \\
(n=162)\end{array}$ & $\begin{array}{l}54.8 \% \\
(n=223)\end{array}$ \\
\hline $\begin{array}{l}\text { Increased use of positive parenting } \\
\text { skills }\end{array}$ & $\begin{array}{l}0 \% \\
(\mathrm{n}=0)\end{array}$ & $\begin{array}{l}6.7 \% \\
(n=27)\end{array}$ & $\begin{array}{l}38.7 \% \\
(n=157)\end{array}$ & $\begin{array}{l}54.7 \% \\
(n=222)\end{array}$ \\
\hline $\begin{array}{l}\text { Increased confidence in effective } \\
\text { parenting }\end{array}$ & $\begin{array}{l}0.2 \% \\
(\mathrm{n}=1)\end{array}$ & $\begin{array}{l}9.4 \% \\
(n=38)\end{array}$ & $\begin{array}{l}40.1 \% \\
(n=163)\end{array}$ & $\begin{array}{l}50.2 \% \\
(n=204)\end{array}$ \\
\hline
\end{tabular}


Commitment to use knowledge and skills gain from Being a Parent

\begin{tabular}{c|c|}
$0.2 \%$ & $5 \%$ \\
$(n=1)$ & $(n=20)$
\end{tabular}

$55.3 \%$

$(n=225)$
2 Scaling sample parents reported high levels of knowledge, skills and confidence acquisition see Table 2. Despite these high levels of acquisition, TARS KSC mean score for the scaling sample was significantly lower than the benchmarking sample with an effect size suggesting a meaningful difference (Scaling TARS KSC Mean=13.9 (SD=2.1); RCT TARS KSC Mean=14.6 $(\mathrm{SD}=1.6), \mathrm{t}(453)=2.3, \mathrm{p}<0.05, \mathrm{~d}=0.3$.

(1)

\section{Parent reported concerns about child difficulties}

Parent child concerns decreased significantly over time, equivalent to medium effect size (CAMC Total: Time 1 mean=63.4, $\mathrm{SD}=22.3$, Time 2 mean=48.6, $\mathrm{SD}=25.3, \mathrm{t}(338)=9.3$, $\mathrm{p}<0.001, \mathrm{ES} \mathrm{d}=0.6$.), see Table 3, with reductions across all problem categories, except idiosyncratic parental concerns (CAMC Conduct Problem: Time 1 mean=65.7, SD=20.5, Time 2 mean $=50.8, \mathrm{SD}=25.5, \mathrm{t}(197)=7.5, \mathrm{p}<0.001, \mathrm{ES} \mathrm{d}=0.6$; CAMC Relationships: Time 1 mean=59.9, $\mathrm{SD}=15.1$, Time 2 mean $=43.4, \mathrm{SD}=22.9, \mathrm{t}(18)=3.5, \mathrm{p}<0.01, \mathrm{ES} \mathrm{d}=0.8 . \mathrm{CAMC}$ Child Self-Regulation: Time 1 mean $=68.2, \mathrm{SD}=21.2$, Time 2 mean=48.6, $\mathrm{SD}=25.3, \mathrm{t}(62)=$ 5.5, $\mathrm{p}<0.001, \mathrm{ES} \mathrm{d}=0.8$. CAMC Child Emotional Distress: Time 1 mean=55.8, $\mathrm{SD}=26.3$, Time 2 mean $=43.7, \mathrm{SD}=25.0, \mathrm{t}(29)=2.0, \mathrm{p}<0.05, \mathrm{ES} \mathrm{d}=0.5$. CAMC Other: Time 1 mean $=55.9, \mathrm{SD}=25.9$, Time 2 mean=45.2, $\mathrm{SD}=23.3, \mathrm{t}(19), \mathrm{p}=$ n.s. $)$. 
1 Table 3: Scaling Programme child, parenting and parent well-being outcomes

\begin{tabular}{|l|l|l|l|l|l|}
\hline Domain & $\mathrm{N}$ & Mean (SD) & Mean (SD) & Significance & Effect \\
& & Time 1 & Time 2 & $(\mathrm{p})$ & size (d) \\
\hline Parental mental well- & 348 & $20.5(3.5)$ & $22.8(3.8)$ & $<0.001$ & 0.6 \\
\hline Parenting behaviour & 348 & $3.5(0.6)$ & $3.0(0.6)$ & $<0.001$ & 0.9 \\
(PS) & & & & & \\
\hline Concerns about my & 339 & $63.4(22.3)$ & $48.6(25.3)$ & $<0.001$ & 0.6 \\
child (CAMC) & & & & & \\
\hline Parenting Goals (PG) & 310 & $36.9(23.0)$ & $69.1(21.0)$ & $<0.001$ & 1.2 \\
\hline
\end{tabular}

2

3 Benchmarking comparison of confidence intervals between Scaling Programme CAMC Total

4 effect size $(d=0.6,95 \% \mathrm{CI}=0.48-0.76)$ and the equivalent $\mathrm{RCT}$ result $(\mathrm{d}=0.85,95 \% \mathrm{CI}=0.42$ -

5 1.26) showed overlapping confidence intervals suggesting that the magnitude of CAMC

6 improvement between the two samples was not significantly different. CAMC subscales

7 were not used in the benchmark RCT.

8

$9 \quad$ Parenting behaviour

10 Scaling programme parents reported significant improvements in positive parenting

11 behaviour, equivalent to a large effect size, (PS Time 1 mean=3.5, $\mathrm{SD}=0.6$, Time 2

12 mean=3.0, $\mathrm{SD}=0.6, \mathrm{t}(347)=14.1, \mathrm{p}<0.001, \mathrm{ES} \mathrm{d}=0.9)$. Pre-course PS mean exceeded the established mean for clinic referred samples and below cut-off post-course. All three PS

14 subscales significantly improved, ranging from small to medium effect sizes (PS Overreactivity Time 1 mean $=3.0, \mathrm{SD}=0.9$, Time 2 mean $=2.5, \mathrm{SD}=0.8, \mathrm{t}(328)=10.6, \mathrm{p}<0.001, \mathrm{ES}$ $\mathrm{d}=0.6$; Verbosity Time 1 mean=4.2, $\mathrm{SD}=0.7$, Time 2 mean=3.9, $\mathrm{SD}=0.7, \mathrm{t}(350)=8.4$, 
$1 \mathrm{p}<0.001, \mathrm{ES} \mathrm{d}=0.4 ;$ Laxness Time 1 mean=3.4, $\mathrm{SD}=0.8$, Time 2 mean $=3.0, \mathrm{SD}=0.7$,

$2 \mathrm{t}(330)=10.0, \mathrm{p}<0.001 \mathrm{ES} \mathrm{d}=0.6)$.

3

4 Scaling sample PS Total Score effect size $(d=0.9$ (95\% CI ADD CIs) and equivalent RCT

5 result $(\mathrm{RCT} \mathrm{d}=0.8(95 \% \mathrm{CI}=0.40-1.20)$ had overlapping confidence intervals, indicating no

6 significant differences in the magnitude of improvement between the two samples.

7

$8 \quad$ Parenting goals

9 Parents reported substantial progress towards achieving selected parenting goals, equivalent

10 to a very large effect size (PG Time 1 mean=36.9, $\mathrm{SD}=23.0$, Time 2 mean=69.1, $\mathrm{SD}=21.0$,

$11 \mathrm{t}(309)=-20.9, \mathrm{p}<0.001, \mathrm{ES} d=1.2)$. No RCT benchmarking information was available for

12 comparison.

14 Parental mental well-being

15 Parents starting Scaling Programme Being a Parent courses had a mean level of mental

16 wellbeing below the UK national $25^{\text {th }}$ centile score of 21.5 [29]. Parents' wellbeing

17 significantly improved following the course equivalent to a medium effect size (Scaling

18 SWEMWBS Time 1 Mean=20.5, $\mathrm{SD}=3.5 \mathrm{SWEMWBS}$ Time 2 mean=22.8, $\mathrm{SD}=3.8), \mathrm{t}(347)=-$

19 11.0, $\mathrm{p}<0.001, \mathrm{ES} d=0.6$ ), with the Time 2 mean score similar to the UK national $50^{\text {th }}$

20 percentile. No RCT benchmarking information was available for comparison.

21

22 Discussion

23

24 Large-scale real-world replication provides evidence about the extent to which efficacious

25 interventions can maintain their performance outside of trial environments and without direct 
1 involvement of intervention developers. This study used a quasi-experimental design with

2 embedded benchmarking to evaluate the scaling-up and scaling-out of the Being a Parent

3 course in 15 newly established EPEC sites. Results indicate that the sites were largely able to

4 replicate the demographic reach, parent acceptability and outcomes previously achieved

5 under trial conditions.

6

7 Sites successfully engaged parents experiencing significant levels of social disadvantage, low

8 levels of formal education and home ownership, together with significant levels of

9 unemployment and lone parenthood. The scaling sample had a larger proportion of White

10 British parents and fewer parents with English as a second language, probably reflecting

11 ethnicity differences between the south London area in which the benchmark RCT took place

12 and the population profiles of participating sites. For example, $19.5 \%$ of the UK population

13 (16-65 years) is of Black and Minority Ethnic origin compared with $46.3 \%$ in the RCT area

$14[40,41]$. Overall, the proportion of Black and Minority Ethnic parents participating in both

15 EPEC trial and scaling programme was higher than local and national base rates.

17 Parent course completion (73.5\%) was consistent with mean completion rates across a wide range of profession-led behavioural parent training programmes though lower than the benchmarking trial comparison [42]. Newly established EPEC sites may not immediately achieve course completion rates equivalent to those accomplished under trial conditions but

21 should expect to achieve rates similar profession-led programmes and, in doing so, engage higher proportions of socially disadvantaged parents.

24 Participants reported high levels of acceptability and satisfaction with course content, 25 methods and parent group leadership largely consistent with benchmarking data. They also 
1 reported statistically significant and clinically meaningful improvements in parental

2 wellbeing, parenting behaviour, parenting goal progress and child concerns. Where

3 comparisons allowed, these improvements were largely similar to benchmarking data and

4 consistent with those reported for comparable professionally-delivered interventions [12-14].

\section{$6 \quad$ Limitations}

7

8 Conclusions about the replication at scale of this evidence-based approach should be drawn

9 with a degree of caution. The study's quasi-experimental design inevitably resulted in lower

10 levels of internal validity compared with those typically achieved by randomised designs.

11 The scaling programme consisted of multiple training and implementation components, many

12 of which, though not all, were manualised and monitored for acceptability, fidelity and

13 impact [30]. However, the current study was not designed to assess the relative impact of

14 these multiple components nor site-related factors affecting performance [43].

16 Benchmarking comparisons provided an efficient, pragmatic and low-cost method to

17 compare scaling programme with trial performance. The study made direct benchmarking comparisons of most demographic and acceptability data though not all outcome measures.

19 Ideally, benchmarking would have included the entire battery of RCT measures. This was

20 not possible because of site concerns about undue real world measurement burden and site

21 preference for the inclusion of the SWEMWBS, not used in the previous RCT. The benchmarking relied upon effect sizes derived from only one RCT. Though of high methodological quality, the benchmarking comparison would have been strengthened by

24 access to a larger pool of trial results, narrowing effect size confidence intervals and reducing 25 the likelihood of type 2 errors. 
2 Data completion rates were at least comparable to similar scaling evaluations, possibly

3 assisted by the emphasis on evaluation within each phase of the scaling implementation and

4 the ongoing commitment of local site supervisors and parent group leaders. However,

5 acceptability and impact data were not available for a significant minority of participants and

6 results are therefore may be susceptible to bias [44]. Typical research trial data acquisition

7 methods, such as participants' positive choice to engage in research, financial and altruistic

8 incentivisation, and dedicated research staff, were not available in this real-world evaluation

9 because of cost constraints.

11 The evaluation infrastructure built during the scaling programme means that EPEC is in a

12 strong position to test data quality improvement methods including ongoing training, rapid

13 and continuous site feedback, site-specific data improvement plan and cooperation on

14 evaluation priorities across sites. Improvements in data quality and larger site samples will

15 enable between-site acceptability and outcome comparisons, as well as the future

16 examination of the effectiveness of specific scaling programme components.

18 Conclusion

20 Despite methodological limitations, these findings suggest that it is possible to successfully

21 replicate at scale EPEC Being a Parent, a peer-led, community-based, parenting approach. In

22 maintaining reach, acceptability and impact at scale, this approach has considerable potential

23 to make a significant contribution to improving child and parenting outcomes and, in so

24 doing, reducing the child behaviour treatment gap, particularly for socially disadvantaged 
1 populations. The results also provide sufficient evidence to warrant a large scale, definitive

2 randomised trial of the scaling programme.

3

4 


\section{List of abbreviations}

2

3 NICE - National Institute for Health and Clinical Excellence

4 EPEC - Empowering Parents Empowering Communities

$5 \quad \mathrm{BaP}-$ Being a Parent

6 NHS - National Health Service

$7 \quad$ EBI - Evidence Based Intervention

$8 \quad \mathrm{RCT}-$ Randomised Controlled Trial

9 CAMC - Concern About My Child measure

10 SWEMWBS - Short Warwick Edinburgh Mental Wellbeing Scale

11 PS - Parenting Scale

12 MPG - My Parenting Goals

13 TARS - Treatment Acceptability Rating Scale

14 ITT - Intention To Treat

15

16

17

18

19

20

21

22

23 


\section{Declarations}

3 Ethics approval and consent to participate - No application for ethical approval was required

4 for the service evaluation of the EPEC National Team Scaling Programme. The funding

5 body, NESTA, independently reviewed and approved the aims, objectives and design of the

6 evaluation prior to commencement. All methods were carried out in accordance with relevant

7 guidelines and regulations (Declaration of Helsinki). Informed consent for the service

8 evaluation was obtained from all participants.

9 Consent for publication - Not applicable.

10 Availability of data and materials - The datasets generated and/or analysed during the current

11 study are not publicly available due privacy of participants involved but are available from

12 the corresponding author on reasonable request.

13 Competing interests - CD is the lead developer of EPEC's Being a Parent course. There are

14 no financial conflicts of interest and no other conflicts of interest.

15 Funding - The scaling programme and evaluation were funded by NESTA and the UK

16 Department for Digital Culture, Media and Sport. Matched funding was received by South

17 London and Maudsley NHS Foundation Trust.

18 Authors' contributions - All authors contributed to the study conception and design. Material

19 preparation, data collection and analysis were performed by $\mathrm{JH}, \mathrm{NK}$ and $\mathrm{CD}$. The first draft

20 of the manuscript was written by $\mathrm{JH}$ and all authors commented on and made revisions to

21 previous versions of the manuscript. All authors read and approved the final manuscript.

22 Acknowledgements - The parents who give up their time to deliver EPEC groups are what

23 makes this programme so special. We would also like to acknowledge the hard work of the

2415 new EPEC hubs who have set up sustainable infrastructure to deliver EPEC groups. 


\section{References}

3 1. Polanczyk GV, Salum GA, Sugaya LS, Caye A, Rohde LA. Annual Research Review: A meta-analysis of the worldwide prevalence of mental disorders in children and adolescents. J Child Psychol Psychiatry. 2015;56(3):345-365; doi:10.1111/jcpp.12381.

6 2. Fergusson DM, Horwood J, Ridder EM. Show me the child at seven: the consequences of conduct problems in childhood for psychosocial functioning in adulthood. J Child Psychol Psychiatry. 2005 Aug 1;46(8):837-49; doi:10.1111/j.1469-7610.2004.00387.x.

3. WHO. World report on violence and health. 2002. https://apps.who.int/iris/bitstream/handle/10665/42495/9241545615_eng.pdf?sequence= 1. Accessed 8 June 2021.

4. Powell T, Barber S, Powell T, Parkin E, Long R, Bolton P, et al. Early Intervention, Briefing paper 7647. House Commons Libr [Internet]. 2019. https://researchbriefings.parliament.uk/ResearchBriefing/Summary/CBP-7647. Accessed 22 Jan 2020.

5. WHO. Preventing violence through the development of safe, stable and nurturing relationships between children and their parents and caregivers. 2009. https://apps.who.int/iris/bitstream/handle/10665/44088/9789241597821_eng.pdf?sequen ce $=1$. Accessed 8 June 2021.

6. Mandalia D, Sadler K, Vizard T, Ford T, Goodman A, Goodman R, et al. Mental Health of Children and Young People in England, 2017. NHS Digit. 2018. https://digital.nhs.uk/data-and-information/publications/statistical/mental-health-ofchildren-and-young-people-in-england/2017/2017. Accessed 8 June 2021. 
1 7. Parsonage M, Khan L, Saunders A. Building a better future: the lifetime costs of childhood behavioural problems and the benefits of early intervention. Lond Cent Ment Health. 2014.

8. Children's Society. The good childhood inquiry: health research evidence. London: Children's Society; 2008.

9. Andrade LH, Alonso J, Mneimneh Z, Wells JE, Al-Hamzawi A, Borges G, et al. Barriers to mental health treatment: results from the WHO World Mental Health surveys. Psychol Med. 2014;44(6):1303-1317; doi:10.1017/S0033291713001943.

10. Kazdin AE. Annual Research Review: Expanding mental health services through novel models of intervention delivery. J Child Psychol Psychiatry. 2019;60(4):455-472; doi:10.1111/jcpp.12937.

11. National Institute for Health and Care Excellence (NICE). Antisocial behaviour and conduct disorders in children and young people: recognition and management: Clinical guideline 158. 2013. https://www.nice.org.uk/guidance/cg158. [updated 19 Apr 2017]. Accessed 8 June 2021.

12. Furlong M, McGilloway S, Bywater T, Hutchings J, Donnelly M, Smith SM, et al. Behavioural/cognitive-behavioural group-based parenting interventions for children age 3-12 with early onset conduct problems. Cochrane Libr [Internet]. 2010. http://onlinelibrary.wiley.com/doi/10.1002/14651858.CD008225/full. Accessed 12 June 2016.

13. Reyno SM, McGrath PJ. Predictors of parent training efficacy for child externalizing behavior problems - a meta-analytic review. J Child Psychol Psychiatry. 2006 Jan 1;47(1):99-111; doi:10.1111/j.1469-7610.2005.01544.x. 
1 14. Michelson D, Davenport C, Dretzke J, Barlow J, Day C. Do evidence-based interventions work when tested in the "real world?" A systematic review and metaanalysis of parent management training for the treatment of child disruptive behavior. Clin Child Fam Psychol Rev. 2013;16(1):18-34; doi:10.1007/s10567-013-0128-0.

15. Lindsay G, Strand S, Davis H. A comparison of the effectiveness of three parenting programmes in improving parenting skills, parent mental-well being and children's behaviour when implemented on a large scale in community settings in 18 English local authorities: the parenting early intervention pathfinder (PEIP). BMC Public Health. 2011;11(1):962.

16. Lindsay G, Strand S. Evaluation of the national roll-out of parenting programmes across England: the parenting early intervention programme (PEIP). BMC Public Health. $2013 ; 13(1): 972$.

17. Lindsay G, Totsika V. The effectiveness of universal parenting programmes: the CANparent trial. BMC Psychol. 2017;5(1):35; doi:10.1186/s40359-017-0204-1.

18. Kazdin AE. Addressing the treatment gap: A key challenge for extending evidencebased psychosocial interventions. Behav Res Ther. 2017;88:7-18; doi:10.1016/j.brat.2016.06.004.

19 Day C, Michelson D, Thomson S, Penney C, Draper L. Evaluation of a peer led parenting intervention for disruptive behaviour problems in children: community based randomised controlled trial. BMJ. 2012;344:1-10; doi:10.1136/bmj.e1107.

20 Thomson S, Michelson D, Day C. From parent to 'peer facilitator': a qualitative study of a peer-led parenting programme. Child Care Health Dev. 2015;41(1):76-83; doi:10.1111/cch.12132. 
121 Fortin M, Dionne J, Pinho G, Gignac J, Almirall J, Lapointe L. Randomized controlled trials: do they have external validity for patients with multiple comorbidities? Ann Fam Med. 2006;4(2):104-108.

4

22. Hogue A, Dauber S, Henderson CE. Benchmarking family therapy for adolescent behavior problems in usual care: fidelity, outcomes, and therapist performance differences. Adm Policy Ment Health Ment Health Serv Res. 2017;44(5):626-641; doi:10.1007/s10488-016-0769-7.

23. Spilka MJ, Dobson KS. Promoting the internationalization of evidence-based practice: benchmarking as a strategy to evaluate culturally transported psychological treatments. Clin Psychol Sci Pract. 2015;22(1):58-75; doi:10.1111/cpsp.12092.

24. Aarons GA, Sklar M, Mustanski B, Benbow N, Brown CH. "Scaling-out" evidencebased interventions to new populations or new health care delivery systems. Implement Sci. 2017;12(1):111; doi:10.1186/s13012-017-0640-6.

25. Milat AJ, Bauman A, Redman S. Narrative review of models and success factors for scaling up public health interventions. Implement Sci. 2015;10(1):113; doi:10.1186/s13012-015-0301-6.

26. Schoenwald SK, Hoagwood K. Effectiveness, transportability, and dissemination of interventions: what matters when? Psychiatr Serv. 2001;52(9):1190-1197; doi:10.1176/appi.ps.52.9.1190.

27. Ministry of Housing, Communities and Local Government. English indices of deprivation 2019 [Internet]. 2019. http://imd-bypostcode.opendatacommunities.org/imd/2019. Accessed 8 June 2021. 
1 28. Arnold DS, O'leary SG, Wolff LS, Acker MM. The Parenting Scale: a measure of dysfunctional parenting in discipline situations. Psychol Assess. 1993;5(2):137.

29 Fat LN, Scholes S, Boniface S, Mindell J, Stewart-Brown S. Evaluating and establishing national norms for mental wellbeing using the short Warwick-Edinburgh mental wellbeing scale (SWEMWBS): findings from the health survey for England. Qual Life Res. 2017;26(5):1129-1144.

30 Day, C., Nicoll, J., Harwood, J., Kendall, N., Kearney, L. \& Kirkwood, J. (2020) Transforming chidlren's lives: EPEC scaling programme. NESTA/Department of Digital Culture Media \& Sport. London: South London \& Maudsley NHS Foundation

31. National Research Ethics Service (NRES). Defining research. 2013. www.clahrceoe.nihr.ac.uk/wp-content/uploads/2014/04/defining-research.pdf, accessed 24.01.22

32. Twycross, A., Shorten, A. Service evaluation, audit and research: what is the difference? Evidence-Based Nursing. 2014;17:65-66.

33. Wright CC, Sim J. Intention-to-treat approach to data from randomized controlled trials: a sensitivity analysis. J Clin Epidemiol. 2003;56(9):833-842; doi:10.1016/S0895$\underline{4356(03) 00155-0}$

34 Tabachnick, B.G. and Fidell, L.S. Using multivariate statistics. $6^{\text {th }}$ ed. Boston: Pearson; 2013.

35. Field A. Discovering statistics using IBM SPSS statistics [Internet]. Sage; 2013. https://books.google.co.uk/books?hl=en\&lr=\&id=c0Wk9IuBmAoC\&oi=fnd\&pg=PP2\& $\mathrm{dq}=$ andy+fields+statistics\&ots $=$ LaHpMJ-u0E\&sig=1 ccFWTPuG1QI47nqZg7XScnuEE. Accessed 3 Mar 2017. 
1 36. Self-Brown S, Valente JR, Wild RC, Whitaker DJ, Galanter R, Dorsey S, et al. Utilizing benchmarking to study the effectiveness of parent-child interaction therapy implemented in a community setting. J Child Fam Stud. 2012;21(6):1041-1049; doi:10.1007/s10826-012-9566-4.

37. Smithson M. Correct confidence intervals for various regression effect sizes and parameters: the importance of noncentral distributions in computing intervals. Educ Psychol Meas. 2001;61(4):605-632.

38. Lakens D. Calculating and reporting effect sizes to facilitate cumulative science: a practical primer for t-tests and ANOVAs. Front Psychol. 2013;4:863;

39. Minami T, Serlin RC, Wampold BE, Kircher JC, Brown GJ. Using clinical trials to doi:10.3389/fpsyg.2013.00863.

40. ONS. Census. 2011; https://www.ons.gov.uk (2011). Accessed 3 Mar 2017.

41. Williamson C. Protected characteristics in Southwark JSNA factsheet. Southwark Counc. 2017.

42. Chacko A, Jensen SA, Lowry LS, Cornwell M, Chimklis A, Chan E, et al. Engagement in behavioral parent training: review of the literature and implications for practice. Clin Child Fam Psychol Rev. 2016;19(3):204-215; doi:10.1007/s10567-016-0205-2. service organisations: systematic review and recommendations. Millbank Quarterly. 2004, 82(4), 581-629; doi:10.1111/j.0887-378X.2004.00325.x 
1 44. Rotnitzky A, Wypij D. A note on the bias of estimators with missing data. Biometrics.

2 1994;1163-1170. 


\section{Supplementary Files}

This is a list of supplementary files associated with this preprint. Click to download.

- Appendix1EPECProgrammeTheoryPDF.pdf

- Appendix2AnalysisforsystematicbiasofscalingprogrammeparticipantnonrespondersPDF.pdf 